\title{
GROUPOÏDE D'HOMOTOPIE D'UN FEUILLETAGE RIEMANNIEN ET RÉALISATION SYMPLECTIQUE DE CERTAINES VARIÉTÉS DE POISSON
}

\author{
FERnando AlCALDE CUESTa
}

\begin{abstract}
The purpose of this paper is to prove the existence of a symplectic realization for a large classe of regular Poisson manifolds with riemannian two dimensional characteristic foliation. To do so, we will show that the homotopy groupoid of a riemannian foliation is locally trivial.
\end{abstract}

\section{Introduction}

Une structure de groupoïde sur un ensemble $\Gamma$ est la donnée de deux applications $\alpha$ (source) et $\beta$ (but) de $\Gamma$ sur une partie $\Gamma_{0}$ (l'espace des unités) de $\Gamma$, d'une multiplication $\mu$ définie sur l'ensemble $\Gamma * \Gamma$ des couples $\left(\gamma_{2}, \gamma_{1}\right) \in \Gamma \times \Gamma$ tels que $\alpha\left(\gamma_{2}\right)=\beta\left(\gamma_{1}\right)$ et d'une involution $\iota: \Gamma \rightarrow \Gamma$ (l'inversion), vérifiant des axiomes généralisant ceux de groupe. Un groupoide $\Gamma$ est de Lie si les données prennent place dans la catégorie des variétés, $\Gamma_{0}$ est une sous-variété séparée de $\Gamma$ et les applications $\alpha$ et $\beta$ sont des submersions ([CDW]). On dit que $\Gamma$ est à fiores connexes, simplement connexes..., si les fibres de a sont connexes, simplement conexes... Un groupoïde de Lie $\Gamma \underset{\beta}{\stackrel{\alpha}{\rightrightarrows}} \Gamma_{0}$ est localement trivial (de fibre $F$ ) si $\alpha$ et $\beta$ sont des fibrés localement triviaux (de fibre $F$ ).

Soit $(M, \mathcal{F})$ une variété feuilletée. Un groupoïde de Lie $\Gamma \underset{\beta}{\stackrel{\alpha}{\rightrightarrows}} \Gamma_{0}$ est une réalisation de $\mathcal{F}$ si $\Gamma_{0}=M$ et l'image de $(\beta, \alpha): \Gamma \rightarrow M \times M$ est la relation d'équivalence $R$ définie par les feuilles de $\mathcal{F}$ ([DH]). Il est bien connu que $\mathcal{F}$ est réalisé par son groupoïde d'holonomie $\mathrm{Hol}(\mathcal{F}) \underset{\beta}{\stackrel{\alpha}{\rightrightarrows}} M$ qui vérifie la propriété universelle suivante ([P]): pour toute réalisation $\Gamma$ de $\mathcal{F}$, el existe une factorisation

$$
\begin{array}{ccc}
\Gamma \stackrel{f}{\longrightarrow} & \operatorname{Hol}(\mathcal{F}) \\
(\beta, \alpha) \searrow & & \swarrow(\beta, \alpha)
\end{array}
$$

$R$. 
Dans la section 1 on décrira la réalisation de $\mathcal{F}$ par son groupoide d'homotopie $\Pi_{1}(\mathcal{F})$. Les $\alpha$-fibres étant simplement connexes, $\Pi_{1}(\mathcal{F})$ est la réalisation universelle de $\mathcal{F}$ de dimension $2 n+m$ immergée dans le groupoïde grossier $M \times M$, où $n=\operatorname{dim} \mathcal{F}$ et $n+m=\operatorname{dim} M$. La relation $R$ (qui n'est pas en général une variété) peut être munie d'une structure de groupoïde qui en fait un sous-groupoïde de $M \times M$. Toute réalisation $\Gamma$ de $\mathcal{F}$ de dimension $2 n+m$ immergée dans $M \times M$ peut donc s'intérpreter comme une désingularisation de $R, \Pi_{1}(\mathcal{F})$ étant la désingularisation universelle.

Le groupoïde d'holonomie d'un feuilletage riemannien est localement trivial d'après [Wi]. La démonstration reste valable pour le groupoïde d'homotopie. Néanmoins, le théorème de structure de Molino permettra d'établir à la section 2 une description détaillée du groupoỉde d'homotopie qui met en évidence certaines différences entre les deux réalisations. En particulier, on démontrera le théorème suivant:

Théorème 1 . Soit $\mathcal{F}$ un feuilletage riemannien sur une variété compacte $M$, le groupoide d'homotopie $\Pi_{1}(\mathcal{F})$ est localement trivial de fibre $\tilde{L}$, où $\tilde{L}$ est le revêtement universel commun des feuilles de $\mathcal{F}$.

Soit $\mathcal{F}$ orientable de dimension 2. D'après le théorème de stabilité de Reeb, $\mathcal{F}$ est soit un fibré en sphères, soit asphérique, i.e., toutes les feuilles de $\mathcal{F}$ sont asphériques (et donc leur revêtement universel commun est $R^{2}$ ). Alors $\Pi_{1}(\mathcal{F})$ est localement trivial de fibre $S^{2}$ ou $R^{2}$.

Un groupoide symplectique $(\Gamma, \sigma) \underset{\beta}{\stackrel{\alpha}{\Rightarrow}} \Gamma_{o}$ est un groupoide de Lie muni d'une forme symplectique $\sigma$ tel que le graphe de la multiplication soit une sous-variété lagrangienne de $(-\Gamma) \times \Gamma \times \Gamma$, où $-\Gamma=(\Gamma,-\sigma)([\mathrm{CDW}]$, [W2]). L'espace des unités $\Gamma_{0}$ est muni d'une structure de Poisson canonique $\Lambda_{0}$ qui fait de $\alpha$ (resp. $\beta)$ un morphisme (resp. un anti-morphisme) de Poisson. On dit que

$$
(\Gamma, \sigma) \underset{\beta}{\stackrel{\alpha}{\rightrightarrows}}\left(\Gamma_{0}, \Lambda_{0}\right)
$$

est une réalisation symplectique de $\left(\Gamma_{0}, \Lambda_{0}\right)([\mathrm{CDW}])$. Le problème de la réalisation symplectique consiste à construire pour une variété de Poisson $(M, \Lambda)$ un groupoïde symplectique dont l'espace des unités est isomorphe à $(M, \Lambda)$.

Soit $\mathfrak{g}^{*}$ le dual d'une algèbre de Lie $\mathfrak{g}$ muni de la structure de Poisson linéaire canonique définie par le crochet induit par celui de $\mathfrak{g}$. Si $G$ est un groupe de Lie d'algèbre de Lie $\mathfrak{g}$, l'action à droite (resp. à gauche) de $G$ sur $T^{*}(G)=G \times \mathfrak{B}^{*}$ est hamiltonienne de moment $\alpha:(g, \xi) \rightarrow \xi$ (resp. $-\beta$, où $\beta:(g, \xi) \rightarrow a d_{g}^{*} \xi$ est l'action coadjointe de $G$ sur $\left.\mathfrak{g}^{*}\right)$. Alors $T^{*}(G) \underset{\beta}{\stackrel{\alpha}{\rightrightarrows}} \mathfrak{g}^{*}$ est un groupoïde symplectique. Le 3 e théorème de Lie exprime le fait que $\mathfrak{g}^{*}$ est l'espace des unités 
d'un groupoide symplectique. En interprétant les variétés de Poisson comme généralisation en dimension infinie des duals d'algèbres de Lie, le problène de la réalisation symplectique est le problème de l'extension du $3 \mathrm{e}$ théorème de Lie au cas non linéaire (cf. [CDW], [DH], [W2]).

La réalisation d'une variété de Poisson par un groupoïde symplectique local a été démontrée dans [CDW]. Par contre, il y a des obstructions à la réalisation symplectique globale ([D1], [D2]). Le but de la section 3 est la résolution du problème de la réalisation symplectique pour les variétés de Poisson $(M, \Lambda)$ compactes riemanniennes de dimension symplectique 2 (i.e., à feuilletage caractéristique $\mathcal{F}_{\Lambda}$ régulier riemannien de dimension 2). Bref, on démontrera le théorème suivant:

Théorème 2. Soit $\Lambda$ une structure de Poisson riemannienne de dimension symplectique 2 sur une variété $M$ compacte. Alors, i) Si $\mathcal{F}_{\Lambda}$ est asphérique, il existe une réalisation symplectique de $(M, \Lambda)$.

ii) Si $\mathcal{F}_{\Lambda}$ est un fibré en sphères, il existe une réalisation symplectique de $(M, \Lambda)$ si et seulement si le volume symplectique des feuilles est constant.

Enfin, tous les groupoïdes considérés dans la suite seront supposés de Lie à fibres connexes et toutes les structures seront de classe $C^{\infty}$.

Ce travail a profité de l'ambiance stimulante du Laboratoire de Géométrie de l'Université de Lyon. Je remercie $M$. Hector pour de multiples remarques. Je lui suis très reconnaissant. Mes remerciements vont aussi aux M.M. Coste, Dazord et Reventós. Enfin je remercie également tous les participants at Juin feuilleté pour des nombreuses discussions.

\section{Rappels. Résultats préliminaires}

A) Le groupoïde d'homotopie d'une variété.

Soit $P(M)$ l'espace des chemins d'un espace topologique $M$ muni de la topologie compacte-ouverte. Le groupoïde d'homotopie $\Pi_{1}(M) \underset{\beta}{\stackrel{\alpha}{\Rightarrow}} M$ est le quotient de $P(M)$ par la relation d'homotopie des chemins. L'application $\alpha$ (resp. $\beta$ ) est définie par $\alpha([\gamma])=\gamma(0)$ (resp. $\beta([\gamma\})=\gamma(1))$, les unités sont les classes des chemins constants (identifiées aux points de $M$ ) et la multiplication $\mu$ (resp. l'inversion $\iota$ ) est celle déduite de la composition (resp. l'inversion) usuelle des chemins.

Si $M$ est une variété, $(\beta, \alpha): \Pi_{1}(M) \rightarrow M \times M$ est un revêtement et on peut donc munir $\Pi_{1}(M)$ d'une structure de variété qui en fait un groupoïde de Lie. Si $q: \tilde{M} \rightarrow M$ est le revetement universel de $M$, l'action diagonale du groupe $A$ des automorphismes de $q$ sur le groupoide grossier $\tilde{M} \times \tilde{M} \underset{\beta}{\stackrel{\alpha}{\rightrightarrows}} \tilde{M}$ est libre et proprement discontinue. La translation par un élément $a$ de $A$ est un 
automorphisme de $\tilde{M} \times \tilde{M}$ au-dessus de a (i.e., dont la restriction à $\tilde{M}$ est a). On en déduit le diagramme commutatif suivant:

$$
\begin{array}{ccc}
\tilde{M} \times \tilde{M} & \stackrel{\alpha}{\rightrightarrows} & \tilde{M} \\
\tilde{q} \downarrow & \stackrel{\alpha}{\rightrightarrows} & \downarrow q \\
\tilde{M} \times{ }_{A} \tilde{M} & \stackrel{\alpha}{\rightrightarrows} & M,
\end{array}
$$

où $\tilde{q}$ est le passage au quotient. Alors $\tilde{M} \times_{A} \tilde{M}$ est muni d'une structure de groupoïde d'unités $M$ pour laquelle le revêtement $\tilde{q}$ est un morphisme de groupoïdes au-dessus de $q$. Il s'ensuit que le groupoïde $\tilde{M} \times{ }_{A} \underset{M}{\tilde{M}} \underset{\beta}{\stackrel{\alpha}{\rightrightarrows}} M$ est localement trivial de fibre $\tilde{M}$. Enfin, l'application

$$
\tilde{M} \times{ }_{A} \tilde{M} \rightarrow \Pi_{1}(\dot{M})
$$

qui envoie $\tilde{q}(\tilde{y}, \tilde{x})$ sur la classe $[q \circ \tilde{\gamma}]$ de la projection par $q$ d'un chemin $\tilde{\gamma}$ d'origine $\tilde{x}$ et d'extremité $\tilde{y}$ est un isomorphisme de groupoïdes. On a donc le lemme suivant:

Lemme 1. $\Pi_{1}(M)$ est un groupoide localement trivial de fibre $\tilde{M}$ le revêtement universel de $M$.

B) Groupoïde d'homotopie d'un feuilletage.

Soit $(M, \mathcal{F})$ une variété feuilletée telle que $\operatorname{dim} \mathcal{F}=n$ et $\operatorname{dim} M=n+m$. Si $X$ est un espace connexe, une application continue $\gamma: X \rightarrow M$ est tangente $\dot{a} \mathcal{F}$ si l'image de $\gamma$ est contenue dans une feuilie de $\mathcal{F}$.

Le groupoide d'homotopie $\Pi_{1}(\mathcal{F})$ de $\mathcal{F}$ est obtenu en remplaçant $P(M)$ par l'espace $\mathcal{P}(\mathcal{F})$ des chemins tangents à $\mathcal{F}$ et la relation d'homotopie de chemins par celle d'honotopie tangente à $\mathcal{F}$ dans la défnition de $\Pi_{1}(M)$ (cf. [CDW] ou [P]). Si l'on veut munir $\Pi_{1}(\mathcal{F})$ d'une structure de variété, il faut d'abord introduire la notion suivante: un tube de chemins tangents $a \mathcal{F}$ est une application différentiable

$$
\Theta: \mathbb{R}^{n+m} \times[0,1] \rightarrow M
$$

telle que: $u \in \mathbb{R}^{n+m}$.

i) $\Theta^{u}: t \in[0,1] \rightarrow \Theta(u, t) \in M$ est un chemin tangent à $\mathcal{F}$, pour tout

ii) Pour $i=0,1, \Theta_{i}: u \in R^{n+m} \rightarrow \Theta(u, i) \in M$ est une carte locale distinguée.

On écrira $\Theta_{i}^{-1}=\left(g_{i}, f_{i}\right): \Theta_{i}\left(\mathbf{R}^{n+m}\right) \rightarrow \mathbf{R}^{n} \times \mathbf{R}^{m}$ l'application réciproque de $\Theta_{i}$, où $f_{i}: U_{i} \rightarrow \mathrm{R}^{m_{2}}$ est une application distinguée relativement à $\mathcal{F}$.

Remarquons que pour tout $\gamma \in \mathcal{P}(\mathcal{F})$, il existe un tube de chemins $\Theta$ tel que $\Theta^{0}=\gamma$. En effet, il existe des cartes distinguées $h_{0}: \mathbf{R}^{n+m} \rightarrow U_{0}$ et 
$h_{1}: \mathbb{R}^{n+m} \rightarrow U_{1}$ telles que $h_{U}(0)=\gamma(0)$ et $h_{1}(0)=\gamma(1)$ et une application continue $y \in \mathbb{R}^{m} \rightarrow \gamma^{y} \in \mathcal{P}(\mathcal{F})$ vérifiant $\gamma^{y}(0)=h_{0}(0, y), \gamma^{y}(1)=h_{1}(0, y)$ et $\gamma^{0}=\gamma$ (cf. [P]). Alors, pour tout $u=(x, y) \in R^{n+m}$, le chemin $\Theta^{u} \in \mathcal{P}(\mathcal{F})$ d'origine $h_{0}(x, y)$ et d'extremité $h_{1}(x, y)$ est obtenu en prolongeant le chemin $\gamma^{y}$ dans les plaques passant par $\gamma^{y}(0)$ et $\gamma^{y}(1)$. On obtient ainsi un tube de chemins $\Theta$ tel que $\Theta^{0}=\gamma$ et $\Theta_{i}=h_{i}, i=0,1$.

Tout tube $\Theta$ définit de façon évidente des applications continues $\theta$ et $\hat{\theta}$ :

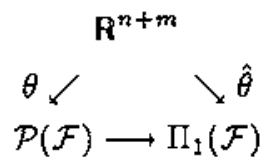

La remarque ci-dessus permet de vérifier aisément que les images des applications $\theta$ (et donc celles des applications $\hat{\theta}$ ) forment une base de la topologie compacte-ouverte sur $\mathcal{P}(\mathcal{F})$ (resp. de la topologie quotient sur $\Pi_{1}(\mathcal{F})$ ). Enfin, en remarquant que pour tout tube $\Theta$, l'application

$$
\begin{array}{lll}
\hat{\theta}\left(\mathbf{R}^{n+m}\right) & \longrightarrow & \mathbf{R}^{n} \times \mathbb{R}^{m} \times \mathbf{R}^{n} \\
\hat{\theta}(u) & \longrightarrow & \left(u, g_{1}\left(\Theta_{1}(u)\right)\right)
\end{array}
$$

est une carte locale, on définit sur $\Pi_{1}(\mathcal{F})$ un atlas et donc une structure de $(2 n+m)$-variété telle que $\alpha$ et $\beta$ sont des submersions, la multiplication $\mu$ est différentiable et l'inversion $t$ est un difféomorphisme. Bref, $\Pi_{1}(\mathcal{F})$ devient un groupoïde de Lie.

Le groupoïde d'homotopie $\Pi_{1}(\mathcal{F})$ est donc une $(2 n+m)$-réalisation de $\mathcal{F}$ à fibres simplernent connexes immergée dans $M \times M$, vérifiant la propriété universelle suivante: pour toute $(2 n+m)$-réalisation $\Gamma \underset{\beta}{\stackrel{\alpha}{\rightrightarrows}} \Gamma_{\mathbb{O}}$ de $\mathcal{F}$ telle que $(\beta, \alpha): \Gamma \rightarrow M \times M$ soit une immersion, il existe un morphisme de groupoïdes de Lie $f: \Pi_{1}(\mathcal{F}) \rightarrow \Gamma$ qui rend commutatif le diagramme suivant:

$$
\begin{gathered}
\Pi_{1}(\mathcal{F}) \stackrel{f}{\longrightarrow} \Gamma \\
(\beta, \alpha) \searrow{ }_{R} /(\beta, \alpha)
\end{gathered}
$$

$R \subset M \times M$ étant la relation d'équivalence définie par $\mathcal{F}$. En effet, pour tout $x \in M, \beta: \alpha^{-1}(x) \rightarrow \beta\left(\alpha^{-1}(x)\right)$ èst un revêtement galoisien de groupe $\Gamma_{x}$ le groupe d'isotropie de $\Gamma$ en $x$. Puisque $\Pi_{1}(\mathcal{F})$ est à fibres simplement connexes, on a naturellement un morphisme continu $f$ qui rend commutatif (1.1). En outre, les applications $(\beta, \alpha)$ de $\Gamma$ et $\Pi_{1}(\mathcal{F})$ dans $M \times M$ étant des immersions, on vérifie sans peine que $f$ est un difféomorphisme local. 
C) Action induite sur $\Pi_{1}(\mathcal{F})$ d'un groupe d'automorphismes de $\mathcal{F}$.

D'abord, tout automorphisme $\phi$ d'une variété feuilletée $(M, \mathcal{F})$ induit un automorphisme $\phi_{\#}$ de $\Pi_{1}(\mathcal{F})$ au-dessus de $\phi$ défini par

$$
\begin{array}{ccc}
\phi_{\# \#}: \Pi_{1}(\mathcal{F}) & \rightarrow & \Pi_{1}(\mathcal{F}) \\
{[\gamma]} & \rightarrow & {[\phi \circ \gamma] .}
\end{array}
$$

En général, l'action d'un groupe $G$ opérant $\operatorname{sur}(M, \mathcal{F})$ comme groupe d'automorphismes induit une action de $G$ sur $\Pi_{1}(\mathcal{F})$ définie par

$$
\begin{array}{cll}
\Pi_{1}(\mathcal{F}) \times G & \rightarrow & \Pi_{1}(\mathcal{F}) \\
([\gamma], g) & \rightarrow & {[\gamma] \cdot g=[\gamma \cdot g]}
\end{array}
$$

qui rend commutatif le diagramme suivant:

$$
\begin{array}{ccc}
\Pi_{1}(\mathcal{F}) \times G & \rightarrow & \Pi_{3}(\mathcal{F}) \\
\alpha \times i d_{G} \downarrow \downarrow \downarrow \times i d_{G} & & \alpha \downarrow \downarrow \\
M \times G & \rightarrow & M
\end{array}
$$

les translations étant des automorphismes de $\Pi_{1}(\mathcal{F})$.

Si l'action de $G$ sur $M$ est libre et propre, il en est de même pour l'action induite sur $\Pi_{1}(\mathcal{F})$ d'après (1.2). Inversement, l'existence d'une section globale de $\alpha$ permet de vérifier que toute action libre et propre d'un groupe $G$ d'automorphismes de $\Pi_{1}(\mathcal{F})$ induit une action libre et propre sur son espace d'unités $M$. Les espaces d'orbites $M / G$ et $\Pi_{1}(\mathcal{F}) / G$ sont des variétés et donc $p: M \rightarrow M / G$ et $\hat{p}: \Pi_{1}(\mathcal{F}) \rightarrow \Pi_{1}(\mathcal{F}) / G$ sont des fibrés principaux de groupe structural $G$.

La structure de groupoïde de Lie sur $\Pi_{1}(\mathcal{F})$ induit sur le quotient $\Pi_{1}(\mathcal{F}) / G$ une structure de groupoïde de Lie d'unités $M / G$. La projection $\tilde{p}$ est un morphisme de groupoides de Lie au-dessus de $p$ qui rend commutatif le diagramme suivant

$$
\begin{array}{ccc}
\Pi_{1}(\mathcal{F}) & \stackrel{\alpha}{\rightrightarrows} & M \\
\downarrow \tilde{p} & \stackrel{\alpha}{\beta} & p \downarrow \\
\Pi_{I}(\mathcal{F}) / G & \underset{\beta}{\rightrightarrows} & M / G .
\end{array}
$$

Lemme 2. $S i \Pi_{1}(\mathcal{F}) \underset{\beta}{\stackrel{\alpha}{\Rightarrow}} M$ est localement trivial de fibre $F$, le groupoide quotient $\Pi_{1}(\mathcal{F}) / G \underset{\beta}{\stackrel{\alpha}{\vec{\beta}}} M / G$ est localement trivial de même fibre.

\section{Démonstration:}

On va montrer que: étant donné un fibré localement trivial $F \rightarrow M \stackrel{\pi}{\rightarrow} B$ équivariant par l'action libre et propre d'un groupe $G$, si l'action induite de $G$ sur $B$ est libre et propre, la submersion $\bar{\pi}: M / G \rightarrow B / G$ obtenue par passage 
au quotient est un fibré localement trivial de fibre $F$ qui rend commutatif le diagramme suivant:

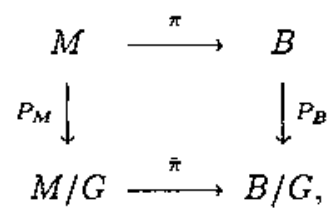

où $P_{M}$ et $P_{B}$ sont les projections naturelles.

Soit $U$ un ouvert contractile de $B / G$; on montrera qu'll trivialise $\bar{\pi}$. En effet, soit $s$ une section de $P_{B}$ au-dessus de $U$. Alors $s(U)$ trivialise $\pi$ et on a donc que $\pi^{-1}(s(U)) \cong F \times s(U) \cong F \times U$. Par ailleurs, l'application $P_{M}: \pi^{-1}(s(U)) \rightarrow(\bar{\pi})^{-1}(U)$ est un difféomorphisme. En effet, son inverse est l'application $(\bar{\pi})^{-1}(U) \rightarrow \pi^{-1}(s(U))$ qui envoie l'orbite $x G$ sur $x \cdot g^{-1}$, où $x \in M$ et $g \in G$ est donné par $\pi(x)=s(\bar{\pi}(x G)) \cdot g$. De plus, celle-ci est différentiable, car il est possible de choisir des sections différentiables de $P_{M}$ au voisinage des points de $(\bar{\pi})^{-1}(U)$. Enfin le lemme est un corollaire de ce qui vient d'être démontré.

On remarque que la construction du groupoide quotient et le lemme 2 restent valables pour toute $(2 n+m)$-réalisation de $\mathcal{F}$.

Soient $q: \tilde{M} \rightarrow M$ un revêtement galoisien de groupe $A, \mathcal{F}$ un feuilletage sur $M$ et $\tilde{\mathcal{F}}=q^{*} \mathcal{F}$ le feuilletage relevé de $\mathcal{F}$ dans $\tilde{M}$. L'action de $A$ laisse invariant $\tilde{\mathcal{F}}$ et donc induit une action libre et proprement discontinue sur $\Pi_{1}(\tilde{\mathcal{F}})$. Alors le quotient $\Pi_{1}(\tilde{\mathcal{F}}) / A$ est muni d'une structure de groupoide d'unités $M=\tilde{M} / A$, le revêtement $\dot{q}: \Pi_{1}(\tilde{\mathcal{F}}) \rightarrow \Pi_{1}(\mathcal{F})$ étant un morphisme de groupoïdes au-dessus de $q$.

Lemme 3. Si $q:(\tilde{M}, \tilde{\mathcal{F}}) \rightarrow(M, \mathcal{F})$ est un revétement galoisien de groupe $A$, le groupoidde quotient $\Pi_{1}(\tilde{\mathcal{F}}) / A$ est isomorphe au groupoïde d'homotopie $\Pi_{1}(\mathcal{F})$.

\section{Démonstration:}

Tout d'abord, le morphisme de groupoides $q_{*}: \Pi_{1}(\tilde{\mathcal{F}}) \rightarrow \Pi_{1}(\mathcal{F})$ induit par $q$ passe au quotient en un morphisme de groupoïdes

$$
\begin{array}{rll}
\phi: \Pi_{1}(\tilde{\mathcal{F}}) / A & \rightarrow & \Pi_{1}(\mathcal{F}) \\
{[\tilde{\gamma}] A} & \rightarrow & q_{\#}[\tilde{\gamma}]=[q \circ \tilde{\gamma}]
\end{array}
$$


au-dessus de l'identité sur $M$ qui rend commutatif le diagramme suivant:

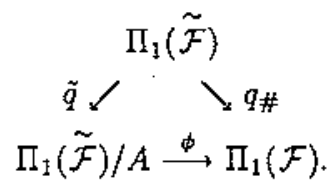

En remarquant que $q_{\#}$ est un revêtement (ce qui est vérifé aisément en restriction aux ouverts coordonnés d'un atlas sur $\Pi_{1}(\mathcal{F})$ construit en procédant comme dans la section $(A)$ ), il suffira de vérifier que $\phi$ est injectif. Étant donnés $\tilde{\gamma}_{1}, \tilde{\gamma}_{2} \in \mathcal{P}(\tilde{\mathcal{F}})$ tels que les chemins projetés $\gamma_{2}=p_{T} \circ \tilde{\gamma}_{1}$ et $\gamma_{2}=p_{T} \circ \tilde{\gamma}_{2}$ soient homotopes dans une feuille de $\mathcal{F}$, lhomotopie entre $\gamma_{1}$ et $\gamma_{2}$ se relève en une homotopie tangente à $\tilde{\mathcal{F}}$ entre $\tilde{\Upsilon}$ et un chemin $\tilde{\gamma}$ qui se projette sur $\gamma_{2}$. Puisque $\tilde{\gamma}$ et $\tilde{\gamma}_{2}$ se projettent sur le même chemin $\gamma_{2}$, il existe un automorphisme qui envoie $\tilde{\gamma}$ sur $\tilde{\gamma}_{2}$ et donc $\left[\tilde{\gamma}_{1}\right] A=[\tilde{\gamma}] A=\left[\tilde{\gamma}_{2}\right] A$. D'où le lemme.

Remarque 1. Le lemme 3 n'est pas valable en général pour le groupoïde d'holonomie: $\operatorname{Hol}(\tilde{\mathcal{F}}) / A$ et $\operatorname{Hol}(\mathcal{F})$ sont isomorphes si et seulement si tout lacet tangent à $\mathcal{F}$ représentant une classe d'holonomie triviale se relève en un lacet tangent à $\tilde{\mathcal{F}}$. En considérant le diagramme naturel suivant

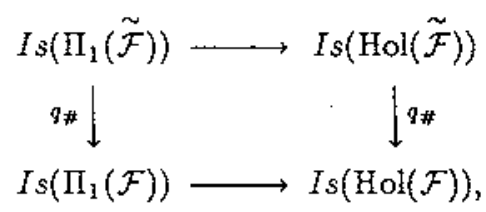

où l'on note $I s(\Gamma)$ le sous-groupoïde d'isotropie $\{\gamma \in \Gamma / \alpha(\gamma)=\beta(\gamma)\}$ d'un groupoïde $\Gamma \underset{\beta}{\stackrel{\alpha}{\rightrightarrows}} \Gamma_{0}$, la condition ci-dessus équivaut à l'injection du morphisme induit $I s\left(\Pi_{1}(\mathcal{F})\right) / q_{\# \#}\left(I_{s}\left(\Pi_{1}(\tilde{\mathcal{F}})\right)\right) \rightarrow I s(\operatorname{Hol}(\mathcal{F})) / q_{\#}(I s(\operatorname{Hol}(\tilde{\mathcal{F}})))$ qui devient donc un isomorphisme de fibrés en groupes sur $M$.

D) Le groupoïde d'homotopie d'une fibration.

Soit $\pi: M \rightarrow B$ un fibré localement trivial de fibre $F$ et groupe structural $G$ défini par un cocycle $\left(\left\{U_{i}\right\},\left\{g_{i j}\right\}\right)$. Soit $E\left(B, \pi_{\#}, \Pi_{1}(F)\right)$ le fibré associé au fibré $M(B, \pi, F)$ et à l'action induite de $G$ sur $\Pi_{1}(F)$.

En considérant $U_{i}$ comme groupoïde trivial, on munit d'abord $\Pi_{1}(F) \times U_{i}$ de la structure de groupoïde produit $\Pi_{1}(\mathcal{F}) \times U_{i} \underset{\beta \times i d}{\stackrel{\alpha \times i d}{\rightrightarrows}} F \times U_{i}$, ce qui permet de défnir sur la somme disjointe $\amalg\left(\Pi_{1}(F) \times U_{i}\right)$ une structure de groupoïde

$$
\coprod\left(\Pi_{1}(F) \times U_{i}\right) \underset{\cup(\beta \times i d)}{\stackrel{\cup(\alpha \times i d)}{\rightrightarrows}} \coprod\left(F \times U_{i}\right)
$$


localement trivial de fibre $\tilde{F}$ le revétement universel de $F$ d'après le lemme 1. Puis en procédant exactement comme au paragraphe (C), on munit $E$ de la structure de groupoïde d'unités $M$ obtenue par passage au quotient:

$$
\begin{array}{ccc}
\amalg\left(\Pi_{1}(F) \times U_{i}\right) & \stackrel{\cup(\alpha \times i d)}{\rightrightarrows} & \amalg\left(F \times U_{i}\right) \\
\pi_{E} \downarrow & \sqcup(\beta \times i d) & \downarrow \pi_{M} \\
E & \stackrel{\alpha}{\rightrightarrows} & M,
\end{array}
$$

$\pi_{E}$ et $\pi_{M}$ étant les projections naturelles. Il s'ensuit que $\underset{\beta}{\stackrel{\alpha}{\Rightarrow}} M$ est localement trivial de fibre $\tilde{F}$. Enfin, l'application

$$
\begin{array}{ccc}
E & \longrightarrow & \Pi_{2}(\pi) \\
\pi_{E}\left(\left[\gamma_{F}\right], b\right) & \longrightarrow & {[\gamma]}
\end{array}
$$

où $\gamma_{F} \in \mathcal{P}(F), b \in U_{i}$ et $\gamma(t)=\pi_{M}\left(\gamma_{F}(t), b\right)$, est un isomorphisme de groupoïdes d'unités $M$. En résumé, on a démontré le lemme suivant:

Lermme 4. Soit $\Pi_{1}(\pi) \underset{\beta}{\stackrel{\alpha}{\rightrightarrows}} M$ le groupoide d'homotopie d'un fibré $\pi: M \rightarrow B$ de fibre $F$ et groupe structural $G$. Alors, i) $\pi_{\#}=\pi \circ \alpha=\pi \circ \beta: \Pi_{1}(\pi) \rightarrow B$ est le $\Pi_{\perp}(F)$-fibré associé au fibré $\pi: M \rightarrow B$ et à l'action de $G$ sur $\Pi_{2}(F)$.

ii) $\Pi_{1}(\pi)$ est localement trivial de fibre le revétement universel de $F$.

\section{Le groupoïde d'homotopie d'un feuilletage riemannien}

Dans ce paragraphe, on appliquera les considérations précédentes à l'étude du groupoide d'homotopie d'un feuilletage riemannien, en suivant les trois étapes classiques d'après les travaux de P. Molino, [M].

A) Feuilletages de Lie.

Soient $\mathfrak{g}$ une algèbre de Lie et $\mathcal{F}$ un $\mathfrak{g}$-feuilletage de Lie sur une variété compacte $M$. D'après le théorème de structure de Fedida $([\mathbf{F}]), \mathcal{F}$ est défini par une fibration équivariante

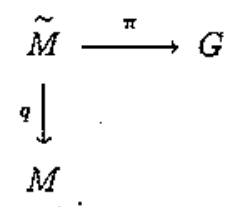

où $G$ est le groupe de Lie connexe et simplement connexe d'algèbre de Lie $\mathfrak{g}$ et $q$ est le revêtement universel de $M$. 
Soit $\tilde{\mathcal{F}}=q^{*} \mathcal{F}$ le feuilletage relevé de $\mathcal{F}$ dans $\tilde{M}$ dont les feuilles sont difféomorphes à la fibre $\tilde{L}$ de $\pi(\tilde{L}$ étant le revêtement universel des feuilles de $\mathcal{F})$. D'après le lemme $4, \Pi_{1}(\tilde{\mathcal{F}})$ est localement trivial de fibre $\tilde{L}$. Si on note $A$ le groupe des automorphismes de $q$, le groupoide quotient $\Pi_{1}(\tilde{\mathcal{F}}) / A$ est donc localement trivial de même fibre. Enfin, le lemme 3 permet d'énoncer la proposition suivante:

Proposition 1. Soit $\mathcal{F}$ un $\mathfrak{b}$-feuilletage de Lie sur une variété compacte $M$. Alors $\Pi_{1}(\mathcal{F})$ est localement trivial de fibre $\tilde{L}$ le revêtement universel des feuilles de $\mathcal{F}$.

\section{B) Feuilletages transversalement parallélisables.}

Soit $\mathcal{F}$ un feuilletage transversalement parallélisable $(T P)$ sur une variété compacte $M$. D'après [M] on sait que:

1) il existe une fibration $\pi: M \rightarrow B$ dite basique dont les fibres sont les adhérences des feuilles de $\mathcal{F}$.

2) il existe une algèbre de Lie $\mathfrak{g}$ telle que le feuilletage $\mathcal{F}_{F}$ induit par $\mathcal{F}$ sur la fibre $F$ de $\pi$ est un $g$-feuilletage de Lie. De plus le groupe structural de la fibration basique $\pi: M \rightarrow B$ admet une réduction au groupe $A=\operatorname{Aut}\left(F, \mathcal{F}_{F}\right)$ des automorphismes de $\left(F, \mathcal{F}_{F}\right)$.

En procédant exactement comme dans la démonstration du lemme 4, le fibré $E\left(B, \pi_{\#}, \Pi_{1}\left(\mathcal{F}_{F}\right)\right)$ associé à la fibration basique et à l'action de $A$ sur $\Pi_{1}\left(\mathcal{F}_{F}\right)$ est muni d'une structure de groupoïde d'unités $M$, localement trivial (de fibre $\tilde{L}$ le revêtement universel commun des feuilles de $\mathcal{F}$ ) et isomorphe à $\Pi_{\mathfrak{1}}(\mathcal{F})$. En résumé, on a le résultat suivant:

Proposition 2. Soit $\mathcal{F}$ un feuilletage $T P$ sur une variété compacte $M$. Soient $\pi: M \rightarrow B$ la fibration basique de $\mathcal{F}$ de fibre $F$ et $\mathcal{F}_{F}=\mathcal{F} / F$. Alors, i) $\pi_{\#}=\pi \circ \alpha=\pi \circ \beta: \Pi_{1}(\mathcal{F}) \rightarrow B$ est le $\Pi_{1}\left(\mathcal{F}_{F}\right)$-fibré associé au fibré $\pi: M \rightarrow B$ et ì l'action du groupe structural $A$ ut $\left(F, \mathcal{F}_{F}\right)$ de $\pi \operatorname{sur} \mathrm{II}_{1}\left(\mathcal{F}_{F}\right)$.

ii) $\Pi_{1}(\mathcal{F})$ est localement trivial de fibre $\tilde{L}$ le revêtement universel des feuilles de $\mathcal{F}$.

\section{C) Feuilletages riemanniens.}

Soit $\mathcal{F}$ un feuilletage riemannien de codimension $m$ sur une variété $M$ compacte, i.e., un feuilletage à bundle-like metric au sens de [R]. Rappelons d'abord que la donnée d'une métrique bundle-like est équivalente à celle d'une $0(m)$ structure transverse ou structure riemannienne sur $(M, \mathcal{F})$ au sens de $[M]$. Soit e $e_{T}\left(M, p_{T}, O(m)\right)$ le fibré des repères transverses orthonormés de $\mathcal{F}_{\text {; }}$ alors if existe sur er un feuilletage $\mathcal{F}_{T}$ appelé le feailletage relevé de $\mathcal{F}$ qui vérifie les propriétés suivantes $([\mathrm{M}])$ : 
i) en restriction à chaque feuille $L_{T} \in \mathcal{F}_{T}, p_{T}$ est un revêtement galoisien sur une feuille $L \in \mathcal{F}$, dont le groupe des automorphisnes est isomorphe aut groupe d'holonomie de $L$.

ii) il existe sur $e_{T}$ une unique connexion $\omega_{T}$ à torsion nulle qui est en plus transverse projetable, i.e., basique sur $\left(\epsilon_{T}, \mathcal{F}_{T}\right)$.

iii) $\mathcal{F}_{T}$ est un feuilletage $T P$ invariant par l'action de $0(m)$ sur $e_{T}$.

iv) La fibration basique $\pi_{T}: e_{T} \rightarrow B_{T}$ de $\mathcal{F}_{T}$ est équivariante par l'action de $0(m)$ et l'espace d'orbites $B_{T} / 0(m)$ de l'action induite sur $B_{T}$ s'identifie à l'ensemble des adhérences des feuilles de $\mathcal{F}$.

D'après (iii), l'action du groupe compact $O(m)$ sur $e_{T}$ induit une action différentiable, libre et propre de $0(m)$ sur $\Pi_{3}\left(\mathcal{F}_{T}\right)$. Le quotient $\Pi_{1}\left(\mathcal{F}_{T}\right) / O(m)$ est donc muni d'une structure de groupoìde d'unités $M$ pour laquelle la projetion $\grave{p}_{T}: \Pi_{1}\left(\mathcal{F}_{T}\right) \rightarrow \Pi_{1}\left(\mathcal{F}_{T}\right) / 0(m)$ devient un morphisme de groupoïdes audessus de $p_{T}: e_{T} \rightarrow M$. D'ailleurs, $\mathcal{F}_{T}$ étant $T P, \Pi_{1}\left(\mathcal{F}_{T}\right)$ est localement trivial de fibre $\tilde{L}$ le revêtement universel des feuilles de $\mathcal{F}_{T}$ d'après la proposition 2 . On en déduit que $\Pi_{3}\left(\mathcal{F}_{T}\right) / 0(m)$ est localement trivial de fibre $\tilde{L}$. Enfin, en remarquant que $\dot{L}$ est le revêtement universel des feuilles de $\mathcal{F}$ d'après (i), on aura démontré le théorème 1 dès qu'on aura montré le lemme suivant:

Lemme 5. Il existe un isomorphisme de groupoïdes

$$
\Phi: \Pi_{1}\left(\mathcal{F}_{T}\right) / 0(m) \rightarrow \Pi_{7}(\mathcal{F})
$$

au-dessus de l'identité sur $M$ qui rend commutatif le diagramme suivant:

$$
\begin{gathered}
\Pi_{1}\left(\mathcal{F}_{T}\right) \\
\bar{p}_{T} \downarrow \\
\Pi_{1}\left(\mathcal{F}_{T}\right) / 0(m) \stackrel{\Phi}{\longrightarrow} \Pi_{1}(\mathcal{F})
\end{gathered}
$$

où $p_{T \#}$ est le morphisme de groupoïdes induit par la projection $p_{T}$.

\section{Démonstration:}

En remarquant que $p_{T \#}$ admet des sections locales différentiables, il suffit de vérifier que le morphisme de groupö̈des

$$
\begin{array}{rll}
\Pi_{1}(\mathcal{F}) / 0(m) & \stackrel{\Phi}{ } & \Pi_{1}(\mathcal{F}) \\
{[\tilde{\gamma}] 0(m)} & \longrightarrow & p_{T \#}[\tilde{\gamma}]=\left\{p_{T} \circ \gamma\right]
\end{array}
$$

obtenu par passage au quotient de $p_{T \#}$ est injectif. Or, puisque $p_{T}$ est un revêtement galoisien en restriction aux feuilles de $\mathcal{F}_{T}$, on peut procéder exactement comme au lemme 3 .

Remarque 2. Les résultats précédents montrent que $\Pi_{1}(\mathcal{F})$ est séparé. 
Remarque 3. Le groupoïde d'holonomie $\operatorname{Hol}(\mathcal{F})$ est isomorphe au groupoïde quotient $\operatorname{Hol}\left(\mathcal{F}_{T}\right) / 0(m)$. En effet, d'après (i) tout lacet tangent à $\mathcal{F}$ qui représente une classe d'holonomie triviale se relève en un lacet tangent à $\mathcal{F}_{T}$ (cf. remarque 1).

Remarque 4. D'après le lemme $5, p_{T \#}: \Pi_{1}\left(\mathcal{F}_{T}\right) \rightarrow \Pi_{1}(\mathcal{F})$ est un fibré principal de groupe structural $0(m)$. Si les adhérences des feuilles de $\mathcal{F}$ sont toutes de même dimension, la description de $\Pi_{1}(\mathcal{F})$ peut être précisée. En remarquant que l'action de $0(m)$ sur $B_{T}$ est localement libre, on a que:

i) la fibration basique $\pi_{T}: e_{T} \rightarrow B_{T}$ passe au quotient en une fibration de Seifert généralisée $\pi: M \rightarrow B$ au-dessus de la variété de Satake $B=B_{T} / 0(m)$ dont la fibre générique $F_{T}$ est la fibre de $\pi_{T}$ (cf. lemme 2),

ii) la fibration $\pi_{T \#}=\pi_{T} \circ \alpha=\pi_{T} \circ \beta: \Pi_{1}\left(\mathcal{F}_{T}\right) \rightarrow B_{T}$ passe au quotient en une fibration de Seifert généralisée $\pi_{\#}=\pi \circ \alpha=\pi \circ \beta: \Pi_{1}(\mathcal{F}) \rightarrow B$ de fibre générique $\Pi_{1}\left(\mathcal{F}_{T} / F_{T}\right)$ le groupoide d'homotopie du feuilletage de Lie $\mathcal{F}_{T} / F_{T}$.

Remarque 5. Soit $\mathcal{F}$ un feuilletage riemannien à métrique bundle-like $g$ sur une variété compacte $M$. Les submersions $\alpha, \beta: \Pi_{1}(\mathcal{F}) \rightarrow M$ induisent un même feuilletage image réciproque $\tilde{\mathcal{F}}$ sur $\Pi_{1}(\mathcal{F})$. Alors il existe une métrique bundle-like $g_{T}$ qui définit une structure riemannienne sur $\left(\Pi_{1}(\mathcal{F}), \tilde{\mathcal{F}}\right)$ compatible avec $\alpha$ et $\beta$. En considérant la décomposition de $T \Pi_{1}(\mathcal{F})$ en la somme directe du fibré tangent à $\widetilde{\mathcal{F}}$ et du fibré orthogonal, si on remplace $g_{T}$ par la métrique $\tilde{g}=\left(\alpha^{*} g+\beta^{*} g\right) \oplus g T$, on voit que:

i) $\alpha, \beta:\left(\Pi_{1}(\mathcal{F}), \tilde{g}\right) \rightarrow(M, g)$ sont des submersions riemanniennes.

ii) l'inversion $\iota:\left(\Pi_{1}(\mathcal{F}), \tilde{g}\right) \rightarrow\left(\Pi_{1}(\mathcal{F}), \tilde{g}\right)$ est une isométrie. Bref, $\left(\Pi_{1}(\mathcal{F}), \tilde{g}\right) \underset{\beta}{\stackrel{\alpha}{\Rightarrow}}$ $(M, g)$ est un groupoïde riemannien au sens de [GGHR].

\section{Réalisation symplectique de variétés de Poisson riemanniennes de dimension symplectique 2}

Les champs hamiltoniens d'une variété de Poisson $(M, \Lambda)$ engendrent un feuilletage de Stefan $\mathcal{F}_{\Lambda}$ appelé le feuilletage caractéristique de $\Lambda$ dont les feuilles admettent une structure symplectique pour laquelle l'inclusion daris $(M, \Lambda)$ est un morphisme de Poisson ([Li], [Wi]). Une variété de Poisson $(M, \Lambda)$ est, régulière $([\mathbf{L i}])$ si $\mathcal{F}_{\Lambda}$ est régulier et la dimension de $\mathcal{F}_{\Lambda}$ est appelée la dimension symplectique de $(M, \Lambda)$. Si de plus $\mathcal{F}_{\Lambda}$ est un feutlletage riemannien, $(M, \Lambda)$ sera dite riemannienne.

Soit $\mathcal{F}$ un feuilletage orientable de dimension 2 sur une variété $M$. Soit $\sigma$ la forme volume des feuilles relativement à une métrique riemannienne sur $M$. Puisque $\mathcal{F}$ est de dimension 2, la forme $\sigma$ est une forme symplectique en restriction à chaque feuille de $\mathcal{F}$. Le couple $(\mathcal{F}, \sigma)$ définit une structure de Poisson $\Lambda$ sur $M$ dont le feuilletage caractéristique $\mathcal{F}_{\Lambda}=\mathcal{F}$. En effet, pour 
toute forme $\zeta \in T^{*}(M)$, soit $\Lambda^{\#}(\zeta)$ le vecteur tangent à $\mathcal{F}$ verifiant

$$
i_{\Lambda} \#(\zeta)=-\zeta
$$

sur $T(\mathcal{F})$, ce qui fournit un morphisme de fibrés vectoriels $\Lambda^{\#}: T^{*}(M) \rightarrow T(M)$ d'image $T(\mathcal{F})$. Soit $\Lambda$ le bitenseur défini par

$$
\Lambda(\zeta, \xi)=\sigma\left(\Lambda^{\#}(\zeta), \Lambda^{\#}(\xi)\right)
$$

pour tout couple $\zeta, \xi \in T^{*}(M)$; son crochet de Schouten $[\Lambda, \Lambda]$ est nul en raison de la dimension de $\mathcal{F}$ et $\mathcal{F}=\mathcal{F}_{A}$ par construction.

Le tenseur de Poisson $\Lambda$ est déterminé par la donnée de $\mathcal{F}$ et de la 2-forme $\sigma$ de type $(0,2)$ relativement à la décomposition $T(M)=N(\mathcal{F}) \oplus T(\mathcal{F})$ de $T(M)$ en la somme directe du fibré tangent $T(\mathcal{F})$ à $\mathcal{F}$ et du fibré orthogonal $N(\mathcal{F})$. On dit que $\sigma$ représente la structure de Poisson $\Lambda$ et on associe à $\Lambda$ la classe de cohomologie feuilletée $[\Lambda]=[\sigma] \in H^{0,2}(M, \mathcal{F})$ (cf. [DH]). On remarque que pour $\mathcal{F}$ fixé, les structures $\Lambda=(\mathcal{F}, \sigma)$ sont classifiées d'après [HMS] par un cône positif de $H^{0,2}(M, \mathcal{F})$.

Soit $\Lambda$ une structure de Poisson riemannienne de dimension symplectique 2 sur une variété compacte $M$ déterminée par la donnée du feuilletage caractéristique $\mathcal{F}$ et d'une 2-forme $\sigma$ de type $(0,2)$ représentant $\Lambda$. Si on note $-M=(M,-\Lambda)$, le groupoïde grossier $\Gamma=(-M) \times M$ est naturellement un groupoide de Poisson (i.e. le graphe de la multiplication est une sous-variété coïsotrope de $(-\Gamma) \times \Gamma \times \Gamma,[D H])$. L'image de l'immersion $(\beta, \alpha): \Pi_{1}(\mathcal{F}) \rightarrow \Gamma$ étant saturée pour le feuilletage caractéristique $T(\mathcal{F}) \oplus T(\mathcal{F})$ de $\Gamma$, on munit $\Pi_{1}(\mathcal{F})$ d'une structure de Poisson $\tilde{\Lambda}$ qui fait de $(\beta, \alpha)$ une immersion de Poisson. La structure de Poisson $\tilde{\Lambda}$ est déterminée par le feuilletage image réciproque $\tilde{\mathcal{F}}=\alpha^{*} \mathcal{F}=\beta^{*} \mathcal{F}$ et la 2 -forme $\tilde{\sigma}=\alpha^{*} \sigma-\beta^{*} \sigma$ vérifiant:

i) $d_{\tilde{\mathcal{F}}} \tilde{\sigma}=0$, où $d_{\tilde{\mathcal{F}}}$ est la différentiation le long des feuilles de $\tilde{\mathcal{F}}$.

ii) $\tilde{\sigma} \wedge \tilde{\sigma}=-2 \alpha^{*} \sigma \wedge \beta^{*} \sigma$ est une forme volume sur les feuilles de $\tilde{\mathcal{F}}$.

Alors la réalisation $\left(\Pi_{1}(\mathcal{F}), \tilde{\Lambda}\right)$ de $\mathcal{F}$ est un groupoïde de Poisson et donc une réalisation de Poisson de $(M, \Lambda)$ au sens de $[\mathrm{DH}]$.

Enfin, on démontrera le théorème 2 , en considérant les deux cas possibles:

i) $\mathcal{F}$ est asphérique et $\Pi_{1}(\mathcal{F})$ est localement trivial de fibre $\mathbf{R}^{2}$.

ii) $\mathcal{F}$ est un fibré $p: M \rightarrow B$ de fibre $S^{2}$.

Dans le cas (i), le groupe structural du fibré $\alpha: \Pi_{1}(\mathcal{F}) \rightarrow M$ admet une réduction à $G L(2, \mathbb{B})$, car $\alpha$ possède une section globale et la fibre est contractile. En considérant la rétraction par déformation sur la section nulle (identifiée à l'espace des unités) qui est une homotopie intégrale relativement à $\tilde{\mathcal{F}}$, on vérifie que la 2 -forme $\tilde{\sigma}$ est $\tilde{\mathcal{F}}$-exacte $\tilde{\sigma}=d_{\tilde{\mathcal{F}}} \lambda$ et donc $d \lambda$ est une extension exacte de la forme symplectique des feuilles. Bref, la structure de Poisson $\tilde{\Lambda}$ 
est exacte $([\mathrm{DH}])$. Soit $N^{*}(\tilde{\mathcal{F}})$ le fibré conormal à $\tilde{\mathcal{F}}$ muni de la forme sym* plectique $\hat{\sigma}=\pi^{*} d \lambda-i^{*} d L$, où $\pi: N^{*}(\tilde{\mathcal{F}}) \rightarrow \Pi_{1}(\mathcal{F})$ est la projection naturelle, $i: N^{*}(\tilde{\mathcal{F}}) \rightarrow T^{*}\left(\Pi_{1}(\mathcal{F})\right)$ est l'inclusion et $L$ est la forme de Liouville sur $T^{*}\left(I_{1}(\mathcal{F})\right)$. Alors

$$
\left(N^{*}(\tilde{\mathcal{F}}), \hat{\sigma}\right) \stackrel{\pi}{\longrightarrow}\left(\Pi_{1}(\mathcal{F}), \tilde{\Lambda}\right) \underset{\beta}{\stackrel{\alpha}{\rightrightarrows}}(M, \Lambda)
$$

est une réalisation symplectique de $(M, \Lambda)$, cf. $[\mathrm{DH}]$.

Dans le cas (ii), on remarque tout d'abord que $\Pi_{1}(\mathcal{F})$ est le produit fibré $S^{2} \times S^{2} \rightarrow M \times_{B} M \stackrel{\dot{p}}{\rightarrow} B$ du fibré $p$ par lui-même. Soit $(\Gamma, \hat{\sigma}) \underset{\beta}{\stackrel{\alpha}{\rightarrow}}(M, \Lambda)$ une réalisation symplectique de $(M, \Lambda)$. Quitte à remplacer $(\Gamma, \hat{\sigma})$ par la réalisation symplectique universelle à fibres simplement connexes ([CDW], $[\mathrm{DH}])$, on a une factorisation:

$$
\begin{gathered}
(\Gamma, \hat{\sigma}) \stackrel{\pi}{\longrightarrow}\left(\Pi_{3}(\mathcal{F}), \tilde{\Lambda}\right) \\
(\beta, \alpha) \searrow \quad \stackrel{\swarrow}{\searrow}(\beta, \alpha) \\
(-M) \times M
\end{gathered}
$$

où $r$ est une submersion surjective qui est un morphisme de Poisson à flbres connexes, séparées et isotropes. La structure de Poisson $\tilde{\Lambda}$ étant canonique, si $\Gamma$ est séparé, $\pi$ est symplectiquement complète au sens de [L]. Dans le cas général, la complétion au sens de [D1] des $\alpha$-fibres de $\Gamma$ ([CDW]) entrainne la complétion des fibres de $\pi$. En résumé, $\pi$ est une réalisation isotrope de Libermann au sens de [D1].

Alors l'obstruction de Dazord à l'existence de réalisations isotropes de Libermann ([DI], [D2]) fournit la condition (ii) du théorème 2. En effet, si on note $\sigma_{b}$ la forme symplectique de la feuille $S_{b}=p^{-1}(b), b \in B$, soit

$$
\begin{aligned}
\operatorname{vol}_{\sigma}: B & \longrightarrow \mathbf{R} \\
b & \longrightarrow \int_{S_{b}} \sigma_{b}
\end{aligned}
$$

la fonction volume symplectique des feuilles. Alors vol ${ }_{\sigma}$ est soit une fonction constante, soit une submersion. Il suffit de vérifier cette propriété dans le cas où $M=S^{2} \times B$ et donc $\Pi_{3}(\mathcal{F})=S^{2} \times S^{2} \times B$. Pour tout 2-cycle entier $c$ de $S^{2} \times S^{2}$, soit $\int_{c} \tilde{\sigma}$ la fonction $b \rightarrow \int_{c_{b}} \tilde{\sigma}_{b}$, obtenue par l'intégration de la forme symplectique $\tilde{\sigma}_{b}=\alpha^{*} \sigma_{b}-\beta^{*} \sigma_{b}$ de $S^{2} \times S^{2} \times\{b\}$ sur le cycle $c_{b}=c \times\{b\}$. Alors, d'après l'obstruction de Dazord le support de la forme $d\left(f_{c} \sigma\right)$ est soit vide, soit $B$. On en déduit que la 1 -forme $d\left(\right.$ vol $\left._{\sigma}\right)$ est soit nulle, soit sans singularités. Enfin, la variété $B$ étant compacte, le volume symplectique des fetilles doit être constant. 
Il faut remarquer que le résultat n'est plus vrai si $B$ n'est pas compacte: $M=S^{2} \times R^{+}$muni de la structure de Poisson $\Lambda$ définie par la fibration triviale sur $\mathrm{R}^{+}$et la 2 -forme $\sigma=t \sigma_{0}$, ò $\sigma_{0}$ est la forme canonique de $S^{2}$ normalisée, possède une réalisation symplectique.

Inversement, si $M=S^{2} \times B$ et le volume symplectique des feuilles vol $\sigma$ est constant $v$, la structure de Poisson $\Lambda$ est présymplectique: la 2-forme $\sigma$ représentant $\Lambda$ est fermée. En fait, il existe d'après [HMS] un difféonorphisme $\phi$ tel que $\phi^{*} \sigma=v \sigma_{0}$. Si la structure de Poisson $\Lambda$ est présymplectique, il en est de même pour la structure $\tilde{\Lambda}$ sur $\Pi_{1}(\mathcal{F})$. Alors

$$
\left(N^{*}(\tilde{\mathcal{F}}), \hat{\sigma}\right) \underset{\rightarrow}{\rightarrow}\left(\Pi_{1}(\mathcal{F}), \tilde{\Lambda}\right) \underset{\beta}{\stackrel{\alpha}{\rightrightarrows}}(M, \Lambda),
$$

oì $\hat{\sigma}=\pi^{*} \hat{\sigma}-i^{*} \mathrm{dL}$, est la réalisation symplectique universelle de $(M, \Lambda)$. Dans le cas général, le principe de recollement des groupoïdes symplectiques ([CDW]) assure l'exsitence d'une réalisation symplectique, of $\left[D_{1}\right]$. 


\section{References}

[CDW] A. Coste , P. Dazord , A. WeInstein, Groupoïdes symplectiques, Public. Dépt. Math. Lyon 1 2/A (1987), 1-62.

[D1] P. DAZORD, Obstruction à un troisième theorème de lie non linéaire pour certaines variétés de Poisson régulières, C.R. Acad. Sc. Paris 306 (1988), 273-278.

[D2] P. DAZORD, Réalisations isotropes de Libermann. Le problème des variables actions-angles dans le cas régulier, Preprint (1989).

[DH] P. DAZORD , G. HECTOR, Sur l'intégrabilité de certaines variétés Poisson régulières, Preprint (1989).

[F] E. FEDIDA, Feuilletages de Lie, Thèse Strasbourg (1973).

[GGHR] A. Gallego, L. Gualandri, G. Hector, A. Reventós, Groupoïdes riemanniens, Preprint "Juin Feuilleté" Lyon (1988).

[HMS] G. Hector , E. MAcías , M. SARALEGuI, Classification des structures de Poisson, Preprint "Juin feuilleté" Lyon (1988).

[L] P. LIBERMANN, Problemes d'équivalence et géométrie symplectique, 3ème Rencontre de Géométrie du Schnepfenried, Astérisque, Soc. Math. France, Paris 107/108 (1982).

[Li] A. LICHNÉRowicz, Les variétés de Poisson et leurs algèbres de Lie, J. Diff. Geom. 12 (1977), 253-300.

[M] P. MoLINo, Géométrie globale des feuilletages riemanniens, Proc. Kon. Nederl. Akad. A. 85 (1) (1982), 45-76.

[P] J. PhILLIPS, The holonomic imperative and the homotopy groupoid of a foliated manifold, Rocky Mountain J. Math. 17 (1987), 151-165.

[R] B. REINHART, Foliated manifolds with bundle-like metrics, Ann of Math 69 (1959), 119-131.

(W1] A. Weinstein, The local structure of Poisson manifolds, J. Diff. Geom. 18 (1983), 523-557.

[W2] A. Weinstein, Symplectic groupoids and Poisson manifolds, Bull. Amer. Math. Soc. 16 (1987), 101-103.

[Wi] H.E. WINKELNKEMPER, The graph of a foliation, Ann. Glob. Analysis and Geometry 1 (1983), 51-75.

\footnotetext{
Dept. de Xeometria e Topoloxía

Universidade de Santiago

15705 Santiago de Compostela

SPAIN
}

Rebut el 31 de Moŗ de 1989 